Please do not remove this page

RMIT

UNIVERSITY

\title{
Wideband RF photonic in-phase and quadraturephase generation
}

Emami, Hossein; Sarkhosh, Niusha; Bui, Lam; Mitchell, Arnan

https://researchrepository.rmit.edu.au/esploro/outputs/9921863374901341/filesAndLinks?institution=61RMIT_INST\&index=null

Emami, H., Sarkhosh, N., Bui, L., \& Mitchell, A. (2008). Wideband RF photonic in-phase and quadraturephase generation. Optics Letters, 33(2), 98-100. https://doi.org/10.1364/OL.33.000098

Document Version: Published Version

Published Version: https://doi.org/10.1364/OL.33.000098

Repository homepage: https://researchrepository.rmit.edu.au

(C) 2008 Optical Society of America

Downloaded On 2023/04/26 21:04:13 +1000

Please do not remove this page 


\title{
Wideband RF photonic in-phase and quadrature- phase generation
}

\author{
Hossein Emami,* Niusha Sarkhosh, Lam Anh Bui, and Arnan Mitchell \\ Microelectronics and Materials Technology Center, School of Electrical and Computer Engineering, RMIT University, \\ GPO BOX 2476V, Melbourne, 3001, Victoria, Australia \\ *Corresponding author: hossein.emami@rmit.edu.au
}

Received August 31, 2007; revised November 20, 2007; accepted November 29, 2007; posted December 3, 2007 (Doc. ID 87097); published January 3, 2008

\begin{abstract}
A photonic implementation of a practical broadband RF Hilbert transformer is demonstrated by using a four-tap transversal system. An almost ideal $90^{\circ}$ phase shift with less than $3 \mathrm{~dB}$ of amplitude ripple has been achieved from 2.4 to $17.6 \mathrm{GHz}$. An efficient method to realize both transformed (quadrature-phase) and reference (in-phase) signal has been achieved by using a coarse wavelength division multiplexing coupler. Extension of the transformer bandwidth and further improvements of its implementation are discussed. (C) 2008 Optical Society of America

OCIS codes: $060.5625,060.2360,070.6020,350.4010$.
\end{abstract}

Photonic processing of microwave signals has received significant attention in the past two decades in an attempt to remove the bandwidth bottlenecks incurred in conventional electronics. This interest has generated the research area known as microwave photonics [1]. Microwave photonics is especially suitable for broadband signal processing. The other advantages include immunity to electromagnetic interference and the ability to provide parallel processing of signals and flexible configurability. Many microwave photonic systems have been proposed and demonstrated over the years, including tunable and shape reconfigurable [2-5] filters and phased-array beam formers [6].

In some applications the signal phase is of critical importance, for instance, vector modulation [7], single-sideband modulation [8], and frequency measurement [9]. In these latter applications, access to both in-phase and quadrature-phase $\left(90^{\circ}\right)$ versions of the signal is necessary. Two orthogonally phased components provide an additional degree of flexibility and versatility for signal processing.

There are several techniques to generate in-phase and quadrature-phased components of an RF signal. The most popular technique utilizes $90^{\circ}$ hybrid couplers. Alternatively, the orthogonal phase component could be generated by using the Hilbert transformation. An electrical implementation of a Hilbert transformer has been demonstrated [10]. It is evident from the reported results that the responses of both the electrical hybrid coupler and the electrical Hilbert transformer were characterized by large phase and amplitude ripple due to significant dispersion existing within the microwave circuits.

A photonic approach to implement an RF Hilbert transformer that exhibits minimum microwave dispersion and achieves close to an ideal response has recently been proposed [11]. In this Letter, a practical implementation of a RF photonic Hilbert transformer has been presented in which much improvement of bandwidth and phase response has been achieved compared with [10]. Furthermore, no network matching is needed, and there is flexibility for dynamically adjusting passband ripple and bandwidth.

The frequency response of an ideal Hilbert transform is $-j \operatorname{sgn}(\omega)$, which exhibits a $90^{\circ}$ frequencyindependent phase shift. The impulse response associated with this transform is $1 /(\pi t)$. This impulse response extends infinitely in time and must be truncated in practice. In addition, the impulse response exhibits negative time; therefore practical implementation of the Hilbert transformer would require a reference that is delayed with respect to the transformed components as shown in Fig. 1 (inset). The continuous time impulse response must also be sampled by discrete taps. An appropriate window function, for instance, a Kaiser windowing function [12], should be applied to the taps to minimize the Gibbs effect caused by truncation. In practice the window used can be manipulated to optimally trade off ripple and bandwidth [11,12]. Having chosen a finite number of discrete taps, exact tap weights are obtained by sampling the windowed Hilbert transform.

To allow tap weights and spacing to be chosen, the Hilbert transformer specifications must be defined.

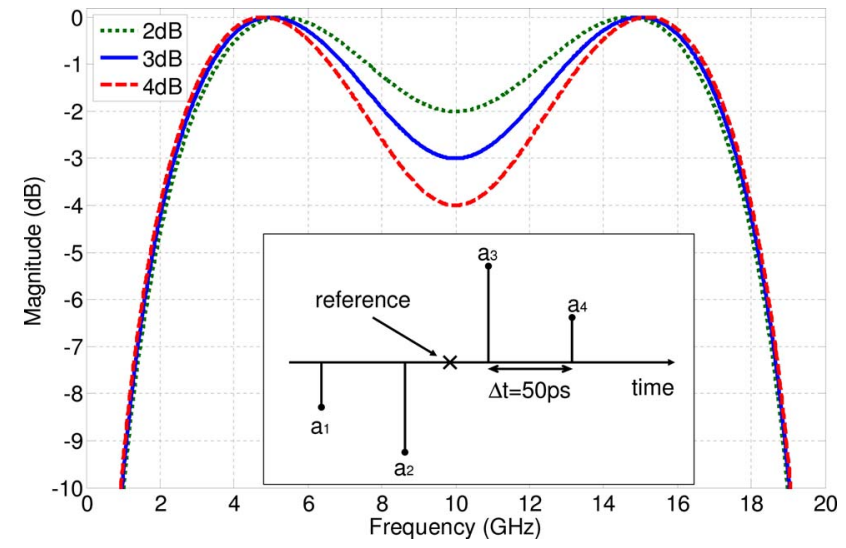

Fig. 1. (Color online) Magnitude of impulse response of a four-tap system. 
The specification for this design were set as bandwidth $2.5-17.5 \mathrm{GHz}$ and passband ripple of amplitude $\leq 3 \mathrm{~dB}$ and phase $\leq 5^{\circ}$. To simplify the demonstration, the design was limited to a transversal system of four taps equally spaced in time. For the bandwidth of $2.5-17.5 \mathrm{GHz}$, the first null of the transversal system was placed at $20 \mathrm{GHz}$, associated with a tap spacing of $1 /(20 \mathrm{GHz})$, or $50 \mathrm{ps}$. The tap weights were adjusted to apply an appropriate window function. In this case, as there were only four taps, the optimization was achieved empirically to achieve the designated ripple and bandwidth. As the impulse response has odd symmetry with the two center taps having the largest amplitude, normalizing the tap weights with respect to the center tap amplitude, the tap weights of the two center taps are always $a_{3}=-a_{2}=1$. The weights of the remaining two taps could be chosen to achieve various levels of passband ripple. The system frequency response was computed by taking the Fourier transform of the impulse response. Figure 1 presents the amplitude response with passband ripples of 2,3 , and $4 \mathrm{~dB}$. The phase response of the three cases of Fig. 1 remained ideal, since the impulse response always has odd symmetry. It is evident from Fig. 1 that the Hilbert transform with $3 \mathrm{~dB}$ of ripple meets the set design specification, which results in $a_{4}=-a_{1}=0.3328$ and $a_{3}=-a_{2}=1$ [11].

Having specified tap weights and spacing, the system was then implemented as shown in Fig. 2. Using a wavelength division multiplexing (WDM) technique, taps $a_{1}-a_{4}$ were associated with optical wavelengths $\lambda_{1}-\lambda_{4}$, respectively. Since the impulse response has odd symmetry, both positive and negative tap coefficients must be implemented.

The wavelengths were divided into two groups of two each. For the first group, $\lambda_{1}$ and $\lambda_{2}$ were combined by using a $3 \mathrm{~dB}$ optical coupler and were modulated by using a Mach-Zehnder modulator (MZM) biased at quadrature with negative slope $\left(\mathrm{V}^{-}\right)$. Similarly, for the second group, $\lambda_{3}$ and $\lambda_{4}$ were combined and modulated with positive slope $\left(\mathrm{V}^{+}\right)[2,4]$. The RF signal was fed equally to both modulators through a broadband Wilkinson power divider. All wavelength channels were then combined. Variable optical lengths (VOL1 and VOL2) were used after the modulators to ensure that the RF signals carried by the wavelengths were subjected to the same delay.

Figures 2(a) and 2(b) show the wavelengths with assigned tap weights. As can be seen, the first wavelength $\left(\lambda_{1}\right)$ had two components. The portion that is shown by a solid line was used as tap $a_{1}$ of the transversal system, while the other portion (dotted line) was used as the system reference. The combined output consisting of four taps is shown in Fig. 2 inset (c). This output was transmitted through a dispersive fiber, where each wavelength experienced a different delay and hence produced taps at different respective times. The dispersed signal was then amplified by using an erbium-doped fiber amplifier (EDFA) to overcome the system loss.

By use of a coarse WDM coupler (CWDM), approximately $90 \%$ of the signal at $\lambda_{1}$ was separated and used as the system reference. Figure 2 insets (d) and (e) show the optical spectrum at Port 1 and Port 2 of the CWDM. At Port 1 all wavelengths $\lambda_{1-4}$ are present, while at Port 2 only the $\lambda_{1}$ component is present. VOL3 and VOL4 were used to time shift the signal at Port 2 to be placed exactly at the middle of the four-tap set before being detected by a photodetector. This became the system reference. This timeshift concept is illustrated in Fig. 2 inset (f). Having obtained the correct tap positions, we tuned the tap amplitudes to $a_{4}=-a_{1}=0.3328$ and $a_{3}=-a_{2}=1$ by carefully adjusting the laser powers.

With a $2.354 \mathrm{~km}$ of single-mode fiber as a dispersive medium with a fiber dispersion of $17 \mathrm{ps} /(\mathrm{nm} \mathrm{km})$, the separation between wavelength channels was computed to be $1.25 \mathrm{~nm}$ to produce a tap spacing of $50 \mathrm{ps}$. We chose $\lambda_{1}=1543.94 \mathrm{~nm}$ to match the splitting wavelength of the CWDM. Wavelengths $\lambda_{2}-\lambda_{4}$ were selected as 1545.19, 1546.44, and $1547.69 \mathrm{~nm}$. Since the dispersion was not exactly linear and nontrivial dispersion was also contributed by other system components, the actual wavelengths for $\lambda_{2}$ and $\lambda_{3}$ were empirically adjusted to 1545.3 and $1546.59 \mathrm{~nm}$.

A vector network analyzer was used to characterize the system response. The vector network analyzer was calibrated with respect to reference output Out 2 and then used to measure the amplitude and phase response of Out 1. These results are presented in Fig.

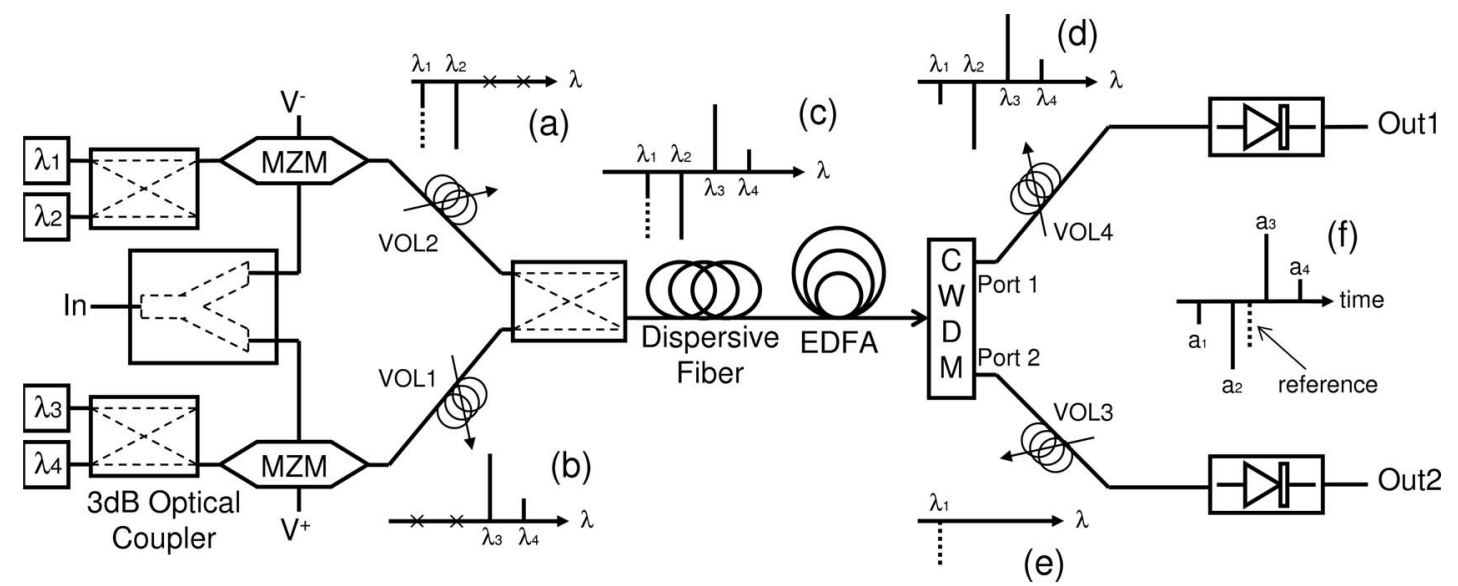

Fig. 2. System implementation. 
3 , together with the calculated responses for the $3 \mathrm{~dB}$ ripple case of Fig. 1 for easy comparison. Excellent agreement between the measurement and simulation is evident. An amplitude variation of $\leq 3 \mathrm{~dB}$ was achieved between 2.4 and $17.6 \mathrm{GHz}$, and an almost ideal $90^{\circ}$ phase response with $\leq 5^{\circ}$ deviation was obtained for all frequencies. An increment of amplitude and phase uncertainty was observed near the system null at $20 \mathrm{GHz}$. This degradation, however, occurred outside the nominal system bandwidth. It was thus concluded that implemented system in fact behaves as theoretically predicted.

Further simplification of the implementation is possible. Replacing the two oppositely biased modulators with a single balanced MZM could eliminate the need for a RF power divider [2,4]. This would also simplify path length equalization and reduce component count and optical splitting losses.

This photonic implementation offers the flexibility of a customized passband response where the pass-
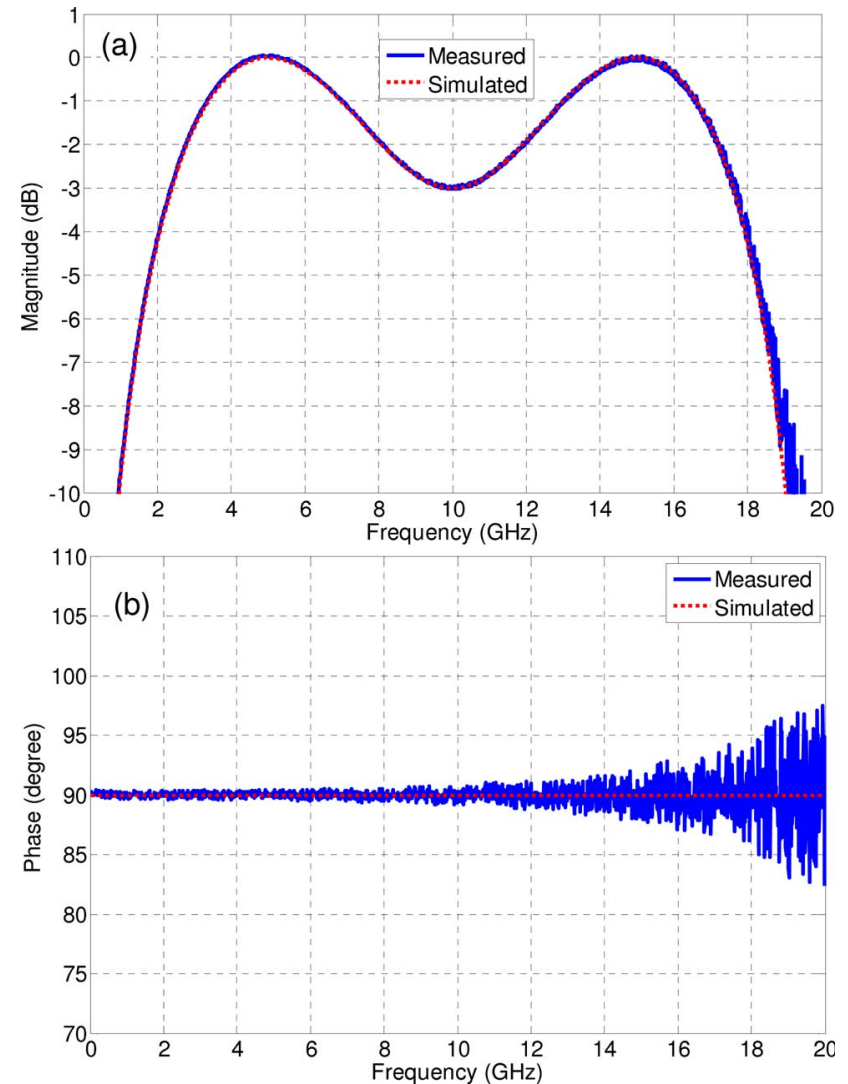

Fig. 3. (Color online) Measured and simulated system response: (a) magnitude, (b) phase. band ripple and the bandwidth can be dynamically configured by changing the tap weights and the wavelength spacing. It also exhibits graceful degradation outside the $3 \mathrm{~dB}$ bandwidth. As the MZMs' input and the photodetectors' output have a $50 \Omega$ impedance, no matching network was needed. It would be desirable to extend the system bandwidth to $2-40 \mathrm{GHz}$ with less than $3 \mathrm{~dB}$ of ripple. Further improvement of bandwidth and ripple could be achieved by employing extra taps as suggested in [11]. Implementations of these improvements are currently under investigation.

In conclusion, a broadband Hilbert transformer was demonstrated by using a photonic transversal signal processing scheme. Efficient use of CWDM enables both transformed (quadrature-phase) and reference (in-phase) signals to be produced. Both system bandwidth and passband ripple can be customized and dynamically configured. No matching network is needed. Suggestions for extension of the bandwidth to $2-40 \mathrm{GHz}$ and further improvements of the implementation were proposed. This Hilbert transformer is suitable for in-phase and quadrature-phase optical processing of broadband microwave signal applications.

\section{References}

1. A. J. Seeds and K. J. Williams, J. Lightwave Technol. 24, 4628 (2006).

2. R. A. Minasian, IEEE Trans. Microwave Theory Tech. 54, 832 (2006).

3. D. B. Hunter and L. V. T. Nguyen, IEEE Trans. Microwave Theory Tech. 54, 900 (2006).

4. J. Capmany, B. Ortega, and D. Pastor, J. Lightwave Technol. 24, 201 (2006).

5. B. Vidal, V. Polo, J. L. Corral, and J. Marti, Electron. Lett. 39, 547 (2003).

6. B. Vidal, T. Mengual, C. Ibanez-Lopez, J. Marti, I. McKenzie, E. Vez, J. Santamaria, F. Dalmases, and L. Jofre, IEEE Photon. Technol. Lett. 18, 1200 (2006).

7. L. A. Bui, A. Mitchell, K. Ghorbani, and T.-H. Chio, IEEE Microw. Wirel. Compon. Lett. 15, 309 (2005).

8. T. Kawanishi and M. Izutsu, IEEE Photon. Technol. Lett. 16, 1534 (2004).

9. L. V. T. Nguyen and D. B. Hunter, IEEE Photon. Technol. Lett. 18, 1188 (2006).

10. C. D. Holdenried, J. W. Haslett, and B. Davies, IEEE Microw. Wirel. Compon. Lett. 15, 303 (2005).

11. H. Emami, L. A. Bui, and A. Mitchell, in Proceedings of Asia Pacific Microwave Photonics Conference (ILCC, 2006), pp. 173-176.

12. J. F. Kaiser and R. W. Schafer, IEEE Trans. Acoust., Speech, Signal Process. ASSP-28, 105 (1980). 